\title{
PROMOCIÓN DE LA SALUD EN EL ÁMBITO ESCOLAR: REFLEXIÓN DESDE LA PERSPECTIVA DE LOS ACTORES DE SU IMPLEMENTACIÓN, EL CASO DE SANTANDER*
}

\author{
Gloria Liliana Rodríguez García** \\ Recibido: Septiembre 1 de 2014 \\ Aprobado: Octubre 10 de 2014
}

\section{RESUMEN}

A partir de la Carta de Ottawa de Promoción a la Salud, El Estado colombiano ha desarrollado un marco normativo extenso que busca garantizar el derecho al desarrollo humano y en consecuencia el derecho a la salud y educación integral. Sin embargo, una vez llega el momento de la implementación de las respectivas políticas públicas, intervienen actores cuya participación en su formulación o diseño fue nula.

Para efectos de este trabajo, se realizó el estudio de la implementación de la promoción de la salud en el ámbito escolar a través de la estrategia Escuelas Saludables desde la perspectiva de los actores responsables en el departamento de Santander, a través de la aplicación de técnicas de investigación cualitativa que permitieron identificar el conocimiento que tienen estos sobre normas, leyes, políticas nacionales, las fortalezas y falencias que identifican los sectores involucrados y los ejecutores de la política territorial, así como sus planteamientos para mejorar el proceso de implementación.

\footnotetext{
* Artículo derivado de la Investigación: "Análisis de la implementación de la política pública de promoción de la salud en el ámbito escolar: escuelas saludables en el departamento de Santander" y aprobada en el 2013 por la Facultad de Finanzas, Gobierno y Relaciones Internacionales de la Universidad Externado de Colombia.

** Nutricionista de la Universidad Industrial de Santander, especialista en Docencia universitaria y Magister en Gobierno y Políticas Públicas, Universidad Externado de Colombia, Columbia University ,N.Y., Profesora de la Universidad Industrial de Santander. Correo electrónico: liliroga@gmail.com
} 
Palabras Claves: Promoción de la salud, implementación, política de salud, salud y bienestar.

\title{
HEALTH PROMOTION AT SCHOOL. SOME CONSIDERATIONS FROM THE PERSPECTIVE OF THOSE IN CHARGE OF ITS IMPLEMENTATION: THE SANTANDER CASE
}

\begin{abstract}
Taking into account the Ottawa Charter for Health Promotion, Colombia has set forth a long legal framework in order to guarantee the right to human development and consequently the right to health and integral education. However, at the time of implementing the corresponding political policies, it is possible to identify the participation of actors who did not contribute to their formulation.

This research focuses on the study of Healthy Schools from the perspective of those in charge of their implementation in the Department of Santander. Techniques of qualitative research permitted to assess their knowledge about rules, laws, national policies, to identify strengthnesses and weaknesses pointed out by the areas involved and by the executors of the territorial policy, and to get acquainted with their proposals in order to improve the process of use of this tool.
\end{abstract}

Key words: Health promotion, implementation, health policy, health and well-being.

\section{INTRODUCCIÓN}

Históricamente se han construido muchos conceptos sobre promoción de la salud, sin embargo, es pertinente contemplar al ser humano como un ser integral y social. En otras palabras, un ser que se afecta tanto por las decisiones individuales, como por los factores ambientales, sociales, culturales y físicos del medio en el que nace, crece, se desarrolla y envejece.

En el país, la promoción de la salud en el ámbito escolar se ha realizado a través de la implementación de la estrategia "Escuelas Saludables", la

1 Denominada así en Colombia, parte de la iniciativa Escuelas Promotoras de la Salud originada en Europa a principios de la década de 1990, para denominar los programas de educación y promoción de la salud en el ámbito escolar. 
cual fue adoptada en 1996 partiendo de los principios y finalidad de la Iniciativa Escuelas Promotoras de la Salud, "La Iniciativa regional de escuelas promotoras de la salud se originó a principios de la década de 1990 a través de múltiples consultas interpaíses que condujeron a su lanzamiento oficial en 1995”. (Ippolito- Shepherd \& Cerqueira, 2003, p.20).

A pesar de la claridad conceptual circunscrita en el proceso de formulación de la estrategia en mención, las investigaciones realizadas sobre el desarrollo de la misma en diferentes periodos desde 1999 hasta el 2012 evidencian que no ha sido totalmente implementada en el territorio nacional y el departamento de Santander. Sin embargo no existen análisis de política pública que permitan conocer desde la perspectiva de los actores las fortalezas y falencias que pudieron afectar este proceso.

Para efectos de esta publicación la metodología de análisis realizada incluyó la aplicación de técnicas de investigación cualitativa que facilitaron el análisis de la política pública desde la perspectiva de estos actores y responsables de su ejecución en el departamento de Santander.

Este estudio cobra importancia porque existe evidencia de la efectividad que tiene la promoción de la salud en el ámbito escolar como estrategia que permite aumentar el bienestar en la infancia y adolescencia, fortalecer las capacidades para alcanzar el desarrollo humano, y actuar sobre los determinantes sociales, culturales, políticos y económicos de la salud desde temprana edad involucrando a la comunidad educativa y a las familias.

\section{MATERIALES Y MÉTODOS}

Se diseñaron y aplicaron dos instrumentos de investigación cualitativa: uno para entrevista semiestructurada y el otro para grupo focal, estos contribuyeron a retroalimentar-desde la perspectiva de los actores- los factores que influyen en el proceso de implementación de la política.

Se aplicaron once entrevistas dirigidas a funcionarios del ámbito departamental -responsables de la implementación- dentro de las cuales tres se aplicaron a funcionarios del sector educación, cinco a funcionarios del sector salud, una profesional coordinadora de proyectos 
de promoción de la salud en el ámbito escolar del Instituto PROINAPSA UIS, un funcionario de una Entidad Promotora de la Salud (EPS) responsable de los procesos de promoción y prevención, un funcionario administrativo de Institución Prestadora de Servicios Pública (IPS pública).

Por otra parte se realizaron dos grupos focales que contaron con la participación de catorce docentes de instituciones educativas públicas del departamento, que en algún momento fueron ejecutores o son ejecutores de la política en la institución educativa.

El procesamiento de la información obtenida incluyó:

1. Transcripción de las grabaciones obtenidas en entrevistas y grupos focales.

2. Categorización de la información teniendo en cuenta las fortalezas, debilidades y oportunidades identificadas por los actores participantes y recomendaciones, respecto a:

- Conocimiento acerca de la política pública de promoción de la salud en el ámbito escolar a través de la Estrategia Escuelas Saludables.

- Conocimiento acerca de los lineamientos que orientan la implementación de esta política en lo local.

- Aspectos de la política pública en mención considerados como fortalezas, debilidades u oportunidades durante su implementación.

- Espacios de concertación o mecanismos para establecer comunicación entre responsables y ejecutores que se han utilizado para coordinar la implementación.

- Conocimiento acerca del seguimiento realizado a la implementación de esta política en el departamento.

- Aspectos para enriquecer el proceso de implementación de esta política en el Departamento.

3. Análisis de la información categorizada y formulación de aportes para el proceso de implementación de la política en el departamento de Santander, los cuales fueron producto de los aportes de los actores participantes del proceso y las reflexiones de la autora de este trabajo de investigación. A continuación se desarrolla un gráfico que permite visibilizar la metodología utilizada para este trabajo de investigación. 
Gráfica 1. Metodología de la investigación

Análisis de la Implementación de la política pública de promoción de la salud en el ámbito escolar: Estrategia Escuelas Saludables en Santander

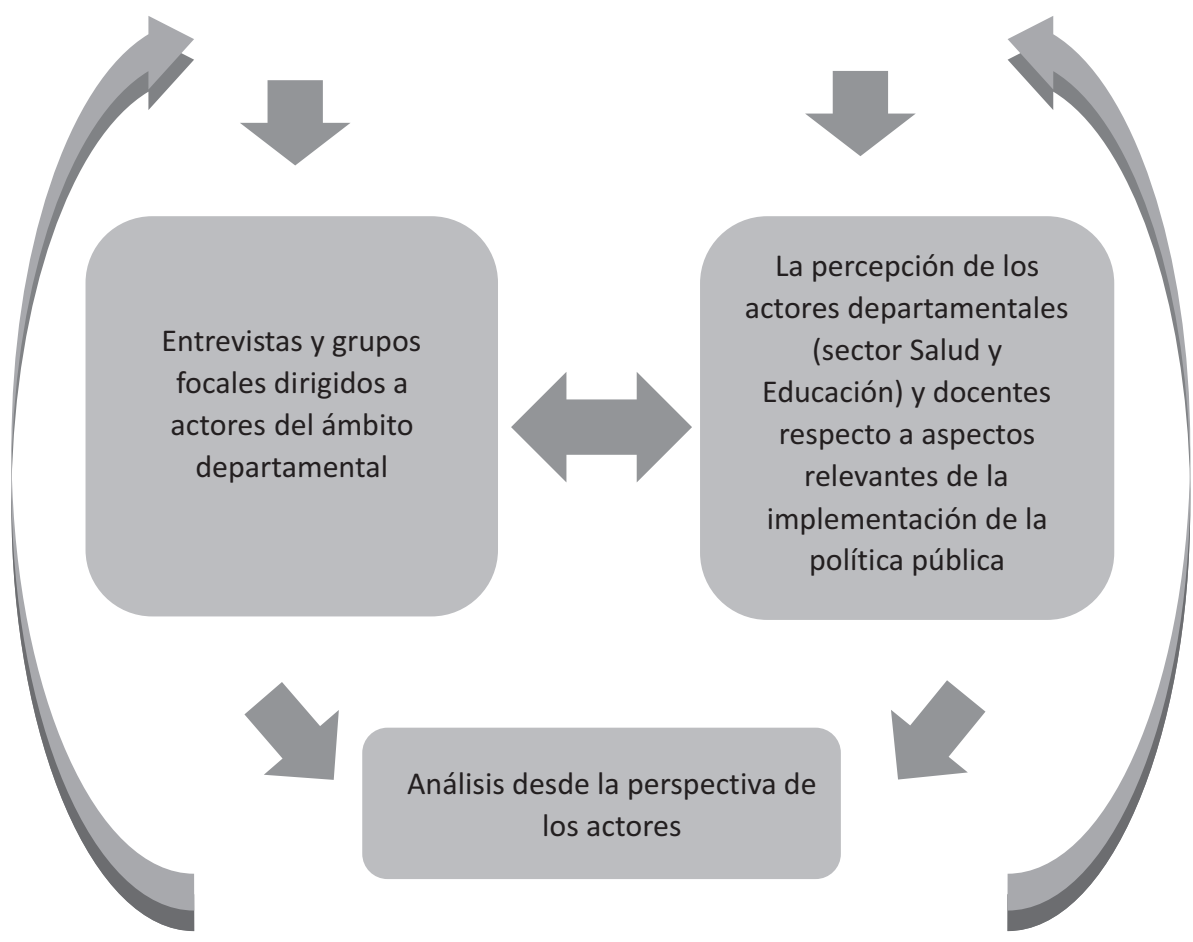

Teoría de la Implementación de las políticas públicas

Construcción propia

\section{LA RELEVANCIA DEL ESTUDIO DE IMPLEMENTACIÓN DESDE LA PERSPECTIVA DE LOS ACTORES}

Es fundamental para el proceso de las políticas públicas indagar sobre las razones por las cuales durante su implementación, sus objetivos 
cambian respecto a lo propuesto durante su construcción, en este sentido es común observar que una política pública no cumple las metas propuestas por razones relacionadas con los ejecutores de la misma, tal como lo expresa Luis F. Aguilar Villanueva:

(...) Más allá del diseño está la implementación de la decisión. Y más allá del presidente y de la legislatura está la compleja red de organizaciones públicas, con sus intereses y sus hábitos, que se encargan de transformar las decisiones en hechos efectivos. (Aguilar et al., 2000, p.31-41)

De acuerdo con esta mirada, se hace pertinente estudiar el proceso de implementación de la estrategia "Escuelas Saludables" concebida como:

(...) una estrategia de promoción y protección de la salud en el ámbito escolar, y un mecanismo articulador de intereses, voluntades y recursos multisectoriales (especialmente aunque no de manera exclusiva, de los sectores salud, educación y ambiente) orientados a aumentar la capacidad y oportunidades de todos los miembros de las comunidades educativas para mejorar la salud, el aprendizaje, la calidad de vida y las oportunidades de desarrollo humano integral y sostenible. (Ministerio de Salud y Protección Social, 2006)

Es importante este estudio si se tiene en cuenta que Colombia fue el primer país de la región en contar con una política pública para el desarrollo de esta estrategia, la cual fue acogida en el año 1997 por el entonces Ministerio de Salud (hoy Ministerio de Salud y de Protección Social) y fue trabajada de manera conjunta con el Ministerio de Educación Nacional, el Instituto Colombiano de Bienestar Familiar (ICBF), la Organización Panamericana de la Salud y la Organización Mundial de la Salud para la elaboración de sus primeros lineamientos. (Ministerio de la Protección Social et al., 2009)

Hoy, a pesar de que esta estrategia es un mecanismo integrador y estratégico para unificar esfuerzos políticos, presupuestales y operativos en torno a la promoción y protección de la salud de la infancia y la adolescencia en el ámbito escolar, no ha sido analizada durante su proceso de implementación. En este análisis de política pública se involucran los responsables y ejecutores de la estrategia como parte fundamental en el proceso de implementación. 
De esta forma, es preciso aclarar que no basta expresar voluntad a través de la promulgación de leyes, normas y diseño de políticas, buena parte de la voluntad de los gobernantes y hacedores de política pública debe verse reflejada en la apuesta por el logro de la efectividad de las políticas y el análisis de los factores que inciden sobre estas.

\section{EL CONCEPTO DE PROMOCIÓN DE LA SALUD}

Históricamente se han construido muchos conceptos sobre promoción de la salud, sin embargo, es pertinente contemplar al ser humano como un ser integral y social. En otras palabras, un ser que se afecta tanto por las decisiones individuales, como por los factores ambientales, sociales, culturales y físicos del medio en el que nace, crece, se desarrolla y envejece. El concepto que se presenta a continuación fue construido durante la primera Conferencia Internacional sobre la Promoción de la Salud reunida en Ottawa el 21 de noviembre de 1986:

La promoción de la salud consiste en proporcionar a los pueblos los medios necesarios para mejorar su salud y ejercer un mayor control sobre la misma. Para alcanzar un estado adecuado de bienestar físico, mental y social un individuo o grupo debe ser capaz de identificar y realizar sus aspiraciones, de satisfacer sus necesidades y de cambiar o adaptarse al medio ambiente. La salud se percibe pues, no como el objetivo, sino como la fuente de riqueza de la vida cotidiana. (Organización Mundial de la Salud, 1986)

Este concepto tiene en cuenta el contexto que rodea a las personas en su vida cotidiana, hace claridad respecto a que existen unas condiciones que deben ser garantizadas y a las capacidades que deben alcanzar las personas para responder ante los cambios y adaptarse a los mismos, reúne los elementos necesarios para construir acciones de promoción de la salud en cualquier ambiente, pues es allí en donde se crea la salud y se teje la vida de las personas.

Por tanto, se trata de un concepto positivo que acentúa los recursos sociales y personales así como las aptitudes físicas. Por consiguiente, dado que el concepto de salud como bienestar trasciende la idea de formas de vida sanas, la promoción de la salud no concierne exclusivamente al sector sanitario. (Organización Mundial de la Salud, 1986). 
La carta de Ottawa contempla cinco áreas fundamentales sobre las cuales se deben diseñar las estrategias de promoción de la salud (Organización Mundial de la Salud, 1986):

1. Construir políticas públicas saludables.

2. Crear entornos favorables (ambientes físicos, sociales, económicos, políticos y culturales).

3. Fortalecer la acción comunitaria.

4. Desarrollar aptitudes personales (estilos de vida).

5. Reorientar servicios de salud.

Las áreas citadas evidencian que el proceso de promoción de la salud debe involucrar la acción de diferentes sectores de gobierno, así como la intervención y la participación de la comunidad. El éxito de la promoción de la salud no depende de manera exclusiva, de las decisiones que el sistema sanitario tome para el diseño y puesta en marcha de políticas públicas:

(...) los gobiernos, los sectores sanitarios y otros sectores sociales y económicos, las organizaciones benéficas, las autoridades locales, la industria y los medios de comunicación. Las gentes de todos los medios sociales están involucradas como individuos, familias y comunidades. (Organización Mundial de la Salud, 1986)

Por otra parte, recae en los individuos la responsabilidad de tomar decisiones acertadas en la vida cotidiana que favorezcan la construcción de hábitos saludables, para lo cual se hace necesario el desarrollo de capacidades que involucren el cuidado de la integridad física y mental de las personas.

Con el fin de aclarar un poco más el concepto de promoción de la salud, cabe acotar que: el concepto de promoción de la salud comúnmente es confundido con el de prevención de la enfermedad, especialmente cuando se trata de diseñar políticas, proyectos o programas relacionados con la salud y el bienestar de la población. No obstante, es fundamental entender que si bien los dos conceptos son muy importantes para alcanzar el bienestar de la población, es preciso diferenciarlos con el fin de optimizar recursos destinados a la salud pública, diseñar estrategias conceptualmente coherentes $\mathrm{y}$, sobre todo, favorecer de manera adecuada a la población beneficiaria de estas políticas. 
El siguiente cuadro (Restrepo et al., 2001) resume los aspectos más importantes que deben tenerse en cuenta al momento de diseñar políticas públicas que involucren procesos de promoción de la salud.

\section{Gráfica 2. Diferencias Conceptuales}

\begin{tabular}{|c|c|c|}
\hline Diferencias & Promoción de la salud & Prevención de la enfermedad \\
\hline Objetivos & $\begin{array}{l}\text { Actuar sobre los determinantes de la } \\
\text { salud, generar opciones saludables para } \\
\text { que la población pueda optar por ellas. }\end{array}$ & $\begin{array}{l}\text { Reducir los factores de riesgo y las } \\
\text { enfermedades, así como proteger } \\
\text { individuos y grupos contra riesgos } \\
\text { específicos. }\end{array}$ \\
\hline $\begin{array}{c}\text { A quién se dirigen } \\
\text { las acciones }\end{array}$ & $\begin{array}{l}\text { Personas y grupos en general con el fin } \\
\text { de realizar procesos relacionados con la } \\
\text { promoción de la salud y actúa sobre } \\
\text { sistemas interdependientes y procesos } \\
\text { sociales, culturales y políticos que } \\
\text { influyen sobre la calidad de vida y la } \\
\text { salud de la población. }\end{array}$ & $\begin{array}{l}\text { Personas y grupos a riesgo de enfermar. } \\
\text { La prevención secundaria a individuos y } \\
\text { grupos en riesgo y enfermos subclínicos } \\
\text { sin manifestaciones obvias de } \\
\text { enfermedad. La prevención terciaria a } \\
\text { enfermos en quienes se quiere prevenir } \\
\text { complicaciones y la muerte. }\end{array}$ \\
\hline $\begin{array}{l}\text { Modelos con los } \\
\text { cuales se } \\
\text { implantan }\end{array}$ & $\begin{array}{l}\text { Sociopolíticos, ecológicos y socio- } \\
\text { culturales, que pretenden generar las } \\
\text { condiciones necesarias para que } \\
\text { individuos y grupos desarrollen } \\
\text { capacidades de actuar sobre los } \\
\text { determinantes sociales de la salud. }\end{array}$ & $\begin{array}{l}\text { Modelos clásicos de salud pública tipo } \\
\text { tamizaje o detección temprana en } \\
\text { poblaciones en riesgo y para el caso de } \\
\text { prevención terciaria prácticas clínicas } \\
\text { preventivas basadas en la evidencia } \\
\text { clínica y rehabilitación. }\end{array}$ \\
\hline $\begin{array}{l}\text { El tipo y el papel } \\
\text { de los } \\
\text { interventores }\end{array}$ & Sociales, políticos y comunitarios. & $\begin{array}{c}\text { Similares para el caso de la prevención } \\
\text { primaria y clínicos para la secundaria y } \\
\text { terciaria. }\end{array}$ \\
\hline Las estrategias & $\begin{array}{c}\text { Promoción de la salud y prevención } \\
\text { primaria presentan algunas similitudes en } \\
\text { cuanto a la utilización de instrumentos } \\
\text { para informar, educar, comunicar y } \\
\text { fortalecer la participación comunitaria, el } \\
\text { empoderamiento y la acción política para } \\
\text { la formulación e implementación de } \\
\text { políticas saludables. }\end{array}$ & $\begin{array}{l}\text { Pruebas discriminatorias en programas } \\
\text { masivos de detección, para separar los } \\
\text { que probablemente tienen una } \\
\text { enfermedad de los que no la tienen y } \\
\text { actuar de manera prioritaria sobre los } \\
\text { primeros o hacer un diagnóstico precoz } \\
\text { para actuar clínicamente }\end{array}$ \\
\hline
\end{tabular}




\section{RESULTADOS SOBRE EL ANÁLISIS DE LOS FACTORES QUE INCIDEN EN LA IMPLEMENTACIÓN DE LA POLÍTICA DE PROMOCIÓN DE LA SALUD EN EL ÁMBITO ESCOLAR: ESCUELAS SALUDABLES, DESDE LA PERSPECTIVA DE LOS RESPONSABLES Y EJECUTORES EN SANTANDER}

\subsection{Grado de conocimiento de la política pública de promoción de la salud en el ámbito escolar a través de la estrategia escuelas saludables}

Pocos de los actores gubernamentales entrevistados reconocen el concepto de la Estrategia Escuelas Saludables como una estrategia integradora a través de la cual se alcanzan procesos de promoción de la salud, la mayoría identifica esta estrategia como las actividades de prevención de la enfermedad, los servicios de salud que deben garantizarse dentro de las instituciones educativas, las campañas de salud, los tamizajes y todas aquellas actividades de obligatorio cumplimiento contempladas en el plan departamental de salud pública. Estos actores manifiestan que cuando se diseñan directrices de política pública desde el nivel nacional y se desconocen las realidades territoriales -o estas directrices no son claras para los funcionarios del territorio- se afecta el proceso de implementación:

Conocemos la norma, pero, nosotros nos demoramos mucho en hacer el rayón en el constructo, entonces, hay una norma que salió pero nos demoramos en interpretarla, para mí, son diez años en la demora, cuando nosotros logremos entender la norma ya pasó o ya lleva como diez años. (Actor gubernamental, sector salud, mayo de 2013).

Los actores que conocen la política mediante la implementación de la Estrategia Escuelas Saludables, reconocen que actualmente no se está implementando en el departamento, debido a que existe desconocimiento sobre los lineamientos diseñados por el Ministerio de Salud y de Protección Social y otros Ministerios desde 2006 y desconocen las diferencias esenciales entre cumplir con indicadores de obligatorio cumplimiento en salud pública (realizando acciones de prevención del riesgo) y el proceso de promoción de la salud:

No hay apropiación, entre otras cosas porque la mayoría de los funcionarios públicos de los sectores responsables (esencialmente salud y educación) desconocen las normas y lineamientos, 
elaborados desde el nivel nacional lo que significa realmente un proceso de promoción de la salud y confunden "promoción de la salud" con "prevención de la enfermedad". (Actor gubernamental, sector salud, mayo de 2013).

Respecto a los docentes de las instituciones educativas conocen la estrategia, no obstante afirman que actualmente no se está implementando y que se siguen aplicando actividades que los docentes lograron entender y que se lograron armonizar dentro del Proyecto Educativo Institucional.

Creo que el hecho de que el sector salud realice tamizajes, vacunación y valoraciones médicas, nutricionales u odontológicas los últimos tres meses de cada año y finalmente desconozcamos cual fue el resultado de los mismos o como están de salud nuestros estudiantes, dice mucho de que no se están realizando procesos de promoción de la salud, trabajamos como islas, salud no se entera realmente de nuestras necesidades y no nos retroalimenta para contribuir en la solución de lo que ellos logran evaluar en nuestras Instituciones Educativas. (Rectora Institución Educativa, mayo de 2013).

Los cinco componentes que forman parte de la estrategia no son recordados por los funcionarios ni por los docentes, los dos componentes que recuerdan con facilidad y que manifiestan que aún se trabajan en algunas instituciones educativas corresponden a:

- Educación para la salud, ya que es el que los docentes reconocen que "saben hacer" en cuanto a la pedagogía. No obstante manifiestan la necesidad de recibir capacitación por parte del sector salud para aclarar conceptos relacionados con hábitos.

- Componente de reorientación de servicios de salud, el cual interpretan como las actividades que se realizan desde el sector salud en la Institución Educativa.

Respecto a esa pregunta,

"no, no recuerdo de manera precisa los componentes, recuerdo que una actividad importante eran las jornadas que coordinábamos con las Universidades y la Secretaría de salud, de vacunación, la toma de peso y talla y cuando se valoraban los niños por odontología, otra cosa importante y que seguimos haciendo es que tratamos de incluir temas de salud en los contenidos de las asignaturas ese fue 
un legado importante que Escuelas Saludables nos dejó". (Docente, mayo 2013).

Finalmente, al indagar sobre el conocimiento de las normas que respaldan la implementación de la estrategia, algunos actores gubernamentales manifiestan no recordarlas, pero afirman que lo más importante es que la estrategia quede contemplada en los planes de desarrollo y en los respectivos planes de salud pública y educación departamentales. De lo contrario no se facilita la implementación "si no se contempla, simplemente no se cumple" (Actor gubernamental, sector salud, mayo de 2013).

Los lineamientos para la aplicación de las Escuelas Saludables se desconocen por parte de los actores y actualmente esta estrategia se interpreta como las actividades de prevención de la enfermedad o acciones de salud pública de obligatorio cumplimiento que son responsabilidad del sector salud.

\subsection{Espacios de concertación o mecanismos definidos para establecer comunicación entre responsables y ejecutores para coordinar el proceso de implementación}

Es un aspecto considerado como esencial para los actores de la política, así mismo lo identifican como una debilidad que afecta el proceso de implementación, ya que no existe un espacio de concertación que permita a los sectores involucrados tomar decisiones relacionadas con la promoción de la salud a través de la estrategia Escuelas Saludables.

Por otra parte, la comunicación entre el sector salud y educación se limita a los acuerdos necesarios para realizar actividades puntuales de salud pública dentro de las instituciones educativas. Los docentes manifiestan desconocer los espacios de concertación en donde consideran que pueden aportar información fundamental para la toma de decisiones sobre la salud escolar.

En este sentido, los actores gubernamentales del sector salud entrevistados manifiestan que la comunicación y puesta en marcha de actividades de mutuo interés entre sectores se dificulta por el bajo interés por parte del sector educación en temas que consideran "de responsabilidad de salud". Por tal razón -manifiestan- se hace necesario empoderar al sector educación sobre la responsabilidad que tienen en los procesos de promoción de la salud en el ámbito escolar. 
Los ejecutores (docentes) sostienen que la comunicación debe ser un proceso participativo que involucre los dos sectores, pero desde el departamento:

La estrategia se debe planear e implementar desde el departamento, no desde los ministerios de salud, educación y medio ambiente. Los programas llegan, aterrizan en nuestras instituciones educativas, no hay concertación para definir prioridades y menos para analizar si tenemos capacidad para cumplir con lo que dicen las políticas, así es muy difícil garantizar que podamos cumplir con todo. De ahí, que debe ser muy importante definir los espacios de concertación en los que se trabaje en promoción de la salud escolar y que nos inviten a participar de ellos, en los espacios en que he participado (Consejos de Política Social) no se habla de este tema. (Directora de Núcleo municipal, sector educación, mayo 2013).

Dentro del análisis realizado se encontró que algunos de los actores del nivel departamental mencionaron que los Ministerios realizan capacitaciones dirigidas a los departamentos para mejorar los procesos de implementación o dar línea técnica, los secretarios departamentales delegan esta participación a contratistas o funcionarios que no están interesados en el tema, tal como lo afirma uno de ellos: "Esto de delegar temas tan importantes a contratistas, es un error, así nunca se logrará posicionar un tema fundamental como lo es el de la promoción de la salud en la escuela" (Funcionario sector salud, Mayo 2013).

Desde el ámbito nacional los actores identifican falencias en el proceso de comunicación ya que se han identificado dificultades al momento de llegar a consenso, en este sentido la toma de decisiones y la orientación dada a la política se convierte en una mirada particular de cada sector sobre el concepto de Escuela Saludable y, de esta forma, se percibe en el ámbito departamental:

Existen dificultades en el consenso requerido desde el nivel nacional, pues la toma de decisiones para orientar las políticas-en este caso la de promoción de la salud en el ámbito escolar- se realiza dependiendo de cuál de los Ministerios se encuentre liderando el tema, esto se expresa de la misma manera en el territorio. (Profesional de proyectos PROINAPSAUIS). 


\subsection{Seguimiento realizado a la implementación de esta política en el departamento de Santander}

Este es un factor sobre el cual -tanto actores gubernamentales como docentes- consideran como falencia pues se desconocen procesos de seguimiento, evaluación y retroalimentación de resultados de la estrategia Escuelas Saludables en el departamento.

En este sentido, los actores gubernamentales manifiestan que durante la implementación de esta estrategia:

No se contemplaron acciones de seguimiento, tampoco fue asignado un responsable o equipo de responsables "de carrera administrativa" en el ámbito departamental que garantizara la "memoria institucional", sobre la implementación de esta política. (Funcionaria sector salud, mayo 2013).

5.4 Recomendaciones dadas por los actores para adecuar la implementación de la política pública de promoción de la salud en el ámbito escolar a través de la Estrategia Escuelas Saludables en Santander

\section{Actores del ámbito departamental:}

1. Es importante que todos los responsables conozcan a fondo la esencia de la Estrategia Escuelas Saludables y que exista claridad sobre las diferencias entre el proceso de promoción de la salud y el de prevención de la enfermedad: “(...) Los y las docentes y los diferentes funcionarios de gobierno deben tener claridad conceptual respecto a estos conceptos" (Profesional de Proyectos, PROINAPSA, mayo 2013).

2. Es fundamental que se conforme un grupo intersectorial que tenga como objetivo analizar la política, definir los responsables.

3. La comunicación entre sectores y entre estos y las instituciones educativas debe darse en un espacio que convoque a todos y que sea exclusivo para tratar temas de promoción de la salud escolar.

4. Las capacitaciones para la implementación de esta política deben involucrar funcionarios de todos los sectores, sobre todo educación y salud que tengan posibilidad de permanecer liderando los procesos (preferiblemente de planta o carrera administrativa). 


\section{Docentes:}

1. La implementación de la estrategia Escuelas Saludables debe darse en la totalidad de las Instituciones Educativas (tanto privadas como estatales) del departamento.

2. Se debe realizar un proceso de capacitación muy completo sobre los lineamientos, entenderlos, reflexionar sobre ellos y que los aportes de los docentes y comunidad educativa en general pueda realizar aportes.

3. Los docentes deben participar de la capacitación constante, los espacios de concertación y recibir directrices claras.

4. Educación y Salud deben reconocer que las instituciones educativas tienen limitaciones y no cuentan con docentes capacitados para garantizar la implementación de Escuelas Saludables y requieren la asesoría constante, el seguimiento, el acompañamiento para adelantar este proceso.

\section{REFLEXIONES DE CIERRE}

\section{La política debe incluir diversos actores sociales y políticos}

En cada departamento y por cada sector debe garantizarse la continuidad del proceso de capacitación en la política de promoción de la salud en el ámbito escolar, una falencia identificada es la alta rotación de actores gubernamentales, especialmente de salud, es por esta razón que se deben tomar medidas al respecto, una de ellas es que deben existir funcionarios de cada sector y docentes de planta con alto conocimiento de la política dispuestos a liderar y garantizar continuidad en el desarrollo de la estrategia desde el nivel municipal y en la institución educativa.

Debe involucrar la participación de toda la comunidad educativa, incluyendo las familias de los escolares, de tal forma que la política se consolide desde lo social; si los actores sociales reconocen que existe un problema serio relacionado con los determinantes sociales de la salud y cómo estos influyen sobre la salud de la infancia y la adolescencia, como los entornos -tanto el escolar como la vivienda-influyen sobre la salud de sus hijos, esta percepción puede lograr la convergencia y el consenso requeridos para alcanzar su sostenibilidad y posicionamiento en los planes de gobierno.

\section{Debe contar con parámetros de seguimiento}

El monitoreo, seguimiento y retroalimentación de los resultados obtenidos a los ejecutores de la política es esencial para la óptima 
implementación y sostenibilidad de la misma. Es necesaria la construcción de un plan para la evaluación de la política, de esta forma medir la efectividad y retroalimentar a la comunidad educativa, garantizar su adhesión y su sostenibilidad. “(...) Por lo general, las evaluaciones buscan responder a ¿cuáles son los efectos atribuibles a la política pública implementada?"(Ordóñez-Matamoros, 2013.p.225).

La política de promoción de la salud es una política de implementación compleja por la transversalidad de los problemas que intenta resolver, la necesidad de involucrar diversos sectores y niveles de gobierno, los múltiples actores sociales responsables de su ejecución y el alto grado de coordinación que requiere. En este sentido se considera fundamental involucrar a los actores en este proceso, desde la comunidad educativa, hasta los actores gubernamentales de los diferentes sectores y niveles de gobierno.

\section{Debe existir coordinación entre diferentes instituciones, sectores y actores sociales, de esta interrelación depende en gran medida el éxito de la política}

Debe quedar definido el espacio de concertación, sectores y la institucionalidad responsable de la participación y toma de decisiones. Si bien existen espacios de concertación departamental, como los consejos de Política Social, en estos aún no se han posicionado el proceso de promoción de la salud en el ámbito escolar, por tal razón es fundamental conformar una mesa de trabajo departamental que facilite el proceso de concertación entre sectores, actores sociales y políticos.

\section{Debe contener lineamientos de actuación con objetivos claros que permitan el cumplimiento y monitoreo de la política}

Existen lineamientos para la implementación de la política de promoción de la salud en el ámbito escolar: Escuelas Saludables, este documento de política pública cuenta con un marco normativo que lo sustenta y que parte desde los acuerdos internacionales hasta las directrices nacionales que favorecen el desarrollo de la estrategia.

Contiene un marco conceptual completo y las directrices respecto a las líneas de acción que deben aplicarse para lograr el proceso de promoción de la salud en el ámbito escolar, estas directrices están dirigidas a resaltar la trascendencia que tiene el que desde la institución educativa se lideren las actividades contempladas en cada línea. 
Pese a que el documento se constituye en el "cómo" la institucionalidad debe poner en marcha lo contemplado desde la normatividad, carece de objetivos claros, con responsabilidades definidas para cada ámbito de gobierno, sector y actor, no está definido el origen del presupuesto que debe ser destinado para la puesta en marcha y las instancias que deben ser utilizadas y/o conformadas para la coordinación de la estrategia, toma de decisiones, el monitoreo y seguimiento.

Lo anterior invita a replantear este documento desde su estructura respecto a los aspectos mencionados, hasta su naturaleza política para lograr la adecuada implementación de la política en el ámbito departamental.

\section{Debe contar con apoyo político, recursos y entorno favorable}

Los líderes políticos del departamento de Santander deben conocer sobre la importancia y efectividad de realizar procesos de promoción de la salud en las instituciones educativas del departamento, de tal forma que se logre implementar la Estrategia Escuelas Saludables como política pública del departamento, la decisión y voluntad política son trascendentales para facilitar la asignación de presupuesto y entorno favorable requeridos.

\section{CONCLUSIONES DE LOS PLANTEAMIENTOS REALIZADOS EN TORNO A MODIFICAR LA NATURALEZA Y EL CONTENIDO TÉCNICO DEL DOCUMENTO LINEAMIENTOS NACIONALES PARA LAAPLICACIÓN DE LAS ESTRATEGIAS: ESCUELAS SALUDABLES Y VIVIENDA SALUDABLE 2006}

De todo lo anterior se deriva que es necesario realizar ajustes al documento actual de política pública para la implementación de Escuelas Saludables especialmente en cuanto a:

- Modificación de la naturaleza del documento y su estructura técnica.

- Delimitación del problema, entendido este proceso no como el fraccionamiento sino como la mirada integral desde el fortalecimiento del desarrollo humano, que es lo que realmente se propone resolver la política de promoción de la salud en el ámbito escolar y construcción de objetivos claros, coherentes con el problema.

- Asignación de responsables por línea de acción, sector responsable, actores responsables, actividades específicas desde la Institución Educativa y desde los sectores de gobierno. 
- Definición del origen de presupuesto para las acciones asignadas a cada sector.

- Definición del espacio de concertación indicado.

- Construcción de un plan de evaluación y monitoreo de la política.

- Inclusión en Plan de Desarrollo, Educación, Plan de Salud Pública departamental y municipal.

- Seleccionar un equipo de funcionarios de los sectores involucrados y los docentes cuya vinculación laboral garantice su permanencia en el proceso y sea el responsable de liderar la implementación y sostenibilidad de la política.

- Diseñar proceso de capacitación dirigida a todos los actores gubernamentales y docentes, en este proceso se debe trabajar en las claridades conceptuales y teóricas que orientan la promoción de la salud como un proceso complejo, multifactorial, transversal, en el cual convergen diversos sectores, actores y niveles de gobierno. También es importante para el proceso de implementación, orientar a los diversos actores sobre los componentes de la política desde su formulación, estrategias de implementación, monitoreo y evaluación.

\section{REFERENCIAS BIBLIOGRÁFICAS}

Aguilar, L. (Ed.). (2000). La implementación de las políticas públicas. México: Porrúa.

Asamblea de Santander. (2008). Plan de Desarrollo departamental 2008-2011. Recuperado de: https://www.uis.edu.co/webUIS/es/ investigacionExtension/documentos/docInteres/pddSantanderIncluyen te.pdf

Asamblea de Santander. (2010). Ordenanza 038 de 2010. Recuperado de: http://www.asambleadesantander.gov.co/ordenanzas2010.php.

Asamblea de Santander. (2012). Ordenanza No. 013 del 23 de abril de 2012. Plan Departamental de Desarrollo del Departamento de Santander. Recuperado de: http://www.asambleadesantander.gov.co/ Doc/Foro/pddsantander.pdf

Asamblea General de Naciones Unidas. (1986). Oficina del Alto Comisionado de las Naciones Unidas para los Derechos Humanos. Recuperado de: http://www2.ohchr.org/spanish/law/desarrollo.htm 
Concejo Municipal de Bucaramanga. Acuerdo No .022 de 2008. Recuperado de: http://www.concejodebucaramanga.gov.co/descargas/ Acuerdo_022_2008.pdf

Congreso de la República de Colombia. (1993). Ley General de Salud 100 de 1993. Recuperado de: http://www.caprecom.gov.co/sitio/ filesnormatividad/Ley-100-de-1993.pdf

Congreso de la República de Colombia. (1994). Ley General de Educación 115. Recuperado de: http://www.mineducacion.gov.co/ 1621/articles-85906_archivo_pdf.pdf

Congreso de la República de Colombia. (2006). Ley 1098 de 2006. Ley de Infancia y Adolescencia. Recuperado de: http://www. secretariasenado.gov.co/senado/basedoc/ley/2006/ley_1098_2006.html

Congreso de la República de Colombia. (2007). Ley 1122. Se modifica Sistema General de la Seguridad Social en Salud y se dictan otras disposiciones. Recuperado de: http:/Www.minsalud.gov.co/ Normatividad/LEY\%201122\%20DE\%202007.pdf

Congreso de la República de Colombia. (2011). Ley 1438 de 2011. Recuperado de: http://www.supersalud.gov.co/supersalud/ LinkClick.aspx?fileticket=zKI3pC4yXco\%3D\&tabid=59\&mid $=614$

Departamento Nacional de Planeación. (2010). Plan Nacional de Desarrollo 2010-2014. Recuperado de: https://www.dnp.gov.co/ PND/PND20102014.aspx

El Pueblo de Colombia. (1991). Banco de la República de Colombia. Constitución Política de Colombia 1991. Recuperado de: http://www.banrep.gov.co/regimen/resoluciones/cp91.pdf.

Gobernación de Santander. (2008). Política departamental de salud. En Santander hacemos país. Recuperado de: http://www.nacionesunidas. org.co/odm/index.php/2013-01-10-20-56-03/finish/6-politicaspublicas/33-politica-departamental-de-salud-en-santander-hacemospais.html

González, M. (2010). Sentencia T-170. Corte Constitucional Colombiana. Recuperado de: http://www.corteconstitucional.gov.co/ RELATORIA/2010/T-170-10.htm 
Ippoilito-Shepherd, J. \& Cerqueira, M.T. (2003). Las Escuelas Promotoras de la Salud en las Américas: una iniciativa regional. Recuperado de: ftp://ftp.fao.org/docrep/fao/006/j0243m/j0243m03.pdf

Ministerio de la Protección Social. (2006). Lineamientos Nacionales para la Aplicación y Desarrollo de las Estrategias de Entornos Saludables: Escuela y Vivienda Saludable. Bogotá: Nuevas Ediciones Ltda.

Ministerio de la Protección Social. (2007). Decreto 3039 de 2007. Decreto 3039 de 2007. Recuperado de: https://www.google.com.co/ search? $\mathrm{q}=$ decreto $+3039 \& \mathrm{aq}=\mathrm{f} \& \mathrm{oq}=$ decreto $+3039 \& \mathrm{aqs}=$ chrome $.0 .57 \mathrm{j}$ $013.9520 \mathrm{j} 0 \&$ sourceid $=$ chrome $\& \mathrm{ie}=\mathrm{UTF}-8$.

Ministerio de la Protección Social. (2008). Resolución 425. Plan de Salud Pública de Intervenciones Colectivas. Recuperado de: http://www.minsalud.gov.co/Normatividad/RESOLUCIÓN\%200425\% 20DE\%202008.pdf

Ministerio de la Protección Social. (2009). Entornos Saludables y Desarrollo Territorial en Colombia: Impulso al logro de los Objetivos de Desarrollo del Milenio con Equidad, Estrategia de Entornos Saludables, Documento 02. Recuperado de: http://www.google.com.co/ url $? \mathrm{sa}=\mathrm{t} \& \mathrm{rct}=\mathrm{j} \& \mathrm{q}=\&$ esrc $=\mathrm{s} \&$ source $=$ web $\& \mathrm{~cd}=1 \& \mathrm{ved}=0 \mathrm{CDAQFjAA}$ \&url $=\mathrm{http} \% 3 \mathrm{~A} \% 2 \mathrm{~F} \% 2 \mathrm{Fwww} . \mathrm{minsalud}$.gov.co\%2FDocumentos $\% 252$ 0y\%2520Publicaciones\%2FEntorno\%2520Saludable $\% 2520 \mathrm{y} \% 2520 \mathrm{D}$ esarrollo\%2520Territorial.pdf\&ei=hJaHUZfCIMrn0gHit4HoBw\&usg $=\mathrm{AFQjCNHEuh}$ dkm7lzn0V8Cp0ZkT0ZkVutw\&sig2=j2sbJHTwOC MiGyKnwNnGDQ\&bvm=bv.45960087,d.dmQ.

Ministerio de Salud y Protección Social. (2012). Plan Decenal de Salud Pública 2012-2021. Recuperado de: http://www.minsalud.gov.co/ plandecenal/Documents/dimensiones/dimensionvidasaludablecondicionesno-transmisibles.pdf

Ordóñez-Matamoros, G. (2013). Manual de análisis y diseño de políticas públicas. Bogotá: Universidad Externado de Colombia.

Revuelta, B. (2007). La implementación de las políticas públicas. Recuperado de: http://dikaion.unisabana.edu.co/index.php/dikaion/ article/view/1379/1515 
Roth, A. (2002). Políticas públicas. Formulación, implementación y evaluación. Bogotá: Panamericana formas e impresos S.A.

Tesis de grado: Análisis de la implementación de la política pública de promoción de la salud en el ámbito escolar: Escuelas Saludables en el Departamento de Santander, Universidad Externado de Colombia, maestría en gobierno y políticas públicas Facultad de Finanzas, Gobierno y Relaciones Internacionales Bogotá d.c., 2013 